Revised manuscript SUBmitted to ApJ

Preprint typeset using $\mathrm{LAT}_{\mathrm{E}} \mathrm{X}$ style emulateapj v. 5/2/11

\title{
IMPULSIVE PHASE CORONAL HARD X-RAY SOURCES IN AN X3.9 CLASS SOLAR FLARE
}

\author{
Qingrong Chen ${ }^{1}$ and Vahé Petrosian ${ }^{1,2}$ \\ 1 Department of Physics and KIPAC, Stanford University, Stanford, CA 94305, USA; qrchen@gmail.com, vahep@stanford.edu and \\ 2 Department of Applied Physics, Stanford University, Stanford, CA 94305, USA. \\ Revised manuscript submitted to ApJ
}

\begin{abstract}
We present analysis of a pair of unusually energetic coronal hard X-ray (HXR) sources detected by the Reuven Ramaty High Energy Solar Spectroscopic Imager (RHESSI) during the impulsive phase of an X3.9 class solar flare on 2003 November 3, which simultaneously shows two intense footpoint (FP) sources. A distinct loop top (LT) coronal source is detected up to $\sim 150 \mathrm{keV}$ and a second (upper) coronal source up to $\sim 80 \mathrm{keV}$. These photon energies, which were not fully investigated in earlier analysis of this flare, are much higher than commonly observed in coronal sources and pose grave modeling challenges. The LT source in general appears higher in altitude with increasing energy and exhibits a more limited motion compared to the expansion of the thermal loop. The high energy LT source shows an impulsive time profile and its nonthermal power law spectrum exhibits soft-hard-soft evolution during the impulsive phase, similar to the FP sources. The upper coronal source exhibits an opposite spatial gradient and a similar spectral slope compared to the LT source. These properties are consistent with the model of stochastic acceleration of electrons by plasma waves or turbulence. However, the LT and FP spectral index difference (varying from 0-1) is much smaller than commonly measured and than that expected from a simple stochastic acceleration model. Additional confinement or trapping mechanisms of high energy electrons in the corona are required. Comprehensive modeling including both kinetic effects and the macroscopic flare structure may shed light on this behavior. These results highlight the importance of imaging spectroscopic observations of the LT and FP sources up to high energies in understanding electron acceleration in solar flares. Finally, we show that the electrons producing the upper coronal HXR source may very likely be responsible for the type III radio bursts at the decimetric/metric wavelength observed during the impulsive phase of this flare.
\end{abstract}

Subject headings: acceleration of particles — Sun: corona — Sun: flares — Sun: radio radiation Sun: X-rays, gamma rays

\section{INTRODUCTION}

It is generally believed that during the impulsive phase of solar flares, electrons are often accelerated to hundreds of $\mathrm{keV}$ (and sometimes to relativistic energies) as a result of energy release by magnetic reconnection. However, the exact mechanisms of particle acceleration are still under much debate (e.g. Miller et al. 1997; Krucker et al. 2008a; Zharkova et al. 2011). These nonthermal electrons are most directly connected to the HXR emission they produce through the well-known bremsstrahlung process (e.g. Lin 1974). Direct detection of HXR sources in the corona is thus of paramount importance to study the energy release and particle acceleration processes. The flare accelerated electrons attached to open field lines will escape from the Sun and produce type III radio bursts and may be detected in situ by space instruments.

HXR imaging observations have shown that for most solar flares, the majority of impulsive phase nonthermal emission comes from the conjugate $\mathrm{FP}$ regions of a closed loop or arcade structure (e.g. Hoyng et al. 1981; Sakao 1994; Saint-Hilaire et al. 2008). This has been interpreted in terms of the collisional thick target model (Brown 1971; Syrovat-Skii \& Shmeleva 1972; Hudson 1972; Petrosian 1973), in which nonthermal electrons move downward from the corona to the chromosphere and radiate most of the HXR emission at the dense FP regions. This simple model with a beam of electrons injected into a coronal loop does not predict a distinct
HXR source from the tenuous corona except at very low energies, say below $\sim 20 \mathrm{keV}$ (e.g. Leach \& Petrosian 1983; Brown et al. 2002). However, within the past two decades distinct coronal HXR sources have been found around the top of a thermal soft X-ray loop in addition to two FP sources, with the first definitive observation made by the Yohkoh/Hard X-ray Telescope (HXT) up to 33-53 keV (Masuda et al. 1994, 1995). Observations of distinct coronal sources have motivated several models in terms of particle acceleration and/or transport effects (see review by Fletcher 1999), assuming different properties of a coronal loop or the accelerated electrons, such as a high loop density (Wheatland \& Melrose 1995; Holman 1996), magnetic field convergence (Fletcher \& Martens 1998, see also Leach 1984), and plasma turbulence (Petrosian \& Donaghy 1999).

Investigations of the Yohkoh and RHESSI flares have shown that coronal HXR emission is a common feature of all flares (e.g. Petrosian et al. 2002; Jiang et al. 2006; Krucker \& Lin 2008c). Analysis of the flares with simultaneously detected LT and FP sources (e.g. Petrosian et al. 2002; Battaglia \& Benz 2006; Shao \& Huang 2009) indicate that in general the LT spectra are much softer than the FP spectra and can be fitted by a relatively steep power law (sometimes plus a lower energy thermal component). This fact, jointly with the finite dynamic range $(\sim 10: 1$ for Yohkoh/HXT and RHESSI), may explain why the LT sources are difficult to detect above $\sim 30 \mathrm{keV}$ when the stronger FP 
sources are in the field of view. Thus in-depth studies of the coronal LT emission largely come from the partially occulted solar flares, in which the more intense FP sources are blocked by the solar limb. In these flares, the HXR spectra are found to be generally much softer than those non-occulted flares (Krucker \& Lin 2008c; Tomczak 2009). On the other hand, some peculiar conditions may yield much stronger coronal bremsstrahlung sources than commonly seen. For example, unusually dense loops can prevent nonthermal electrons reaching the FP regions so that the HXR emission is mainly from the LT region and the loop legs (Wheatland \& Melrose 1995; Veronig \& Brown 2004). There also exist a few large $\gamma$-ray flares, in which the LT bremsstrahlung source is detected up to $200-800 \mathrm{keV}$ during the decay of the HXR and $\gamma$-ray emission and even has harder spectra than the FP sources (Krucker et al. 2008b), interpreted as being due to long time trapping and collisional energy loss of the high energy accelerated electrons in the corona.

Simultaneous analysis of the LT and FP sources extending to high energies would be indispensable for a thorough understanding of acceleration and transport mechanisms. For example, in the stochastic acceleration model, where electrons undergo simultaneous acceleration and pitch angle scattering by plasma waves or turbulence in the coronal radiation region, the spectral difference between the LT and FP sources can serve to determine the energy dependence of the escape time and pitch angle scattering time of the accelerated electrons and thus better constrain theoretical models (Petrosian \& Donaghy 1999; Petrosian \& Chen 2010). This requires high spatial and spectral resolution observations over a wide energy range of both the LT and FP sources. The Yohkoh/HXT has only four broad energy bands spanning from $14-93 \mathrm{keV}$, which greatly limits accurate determination of the spatially resolved spectra. For example, the 1991 January 13 flare (Masuda et al. 1994) has had a significant impact on solar flare research (see review in Fletcher 1999; Krucker et al. 2008a), but to this date the nature of the spectrum of its coronal source still remains somewhat controversial (e.g. Masuda et al. 1994; Alexander \& Metcalf 1997; Masuda et al. 2000; Liu et al. 2010). RHESSI provides HXR imaging spectroscopic observations with a higher resolution and sensitivity extending over a wider energy range to study the fundamental physics of energy release and particle acceleration in solar flares (Lin et al. 2002). This allows for more accurate determination of the spectra of individual HXR sources and better understanding of the underlying physics. RHESSI observations have clearly shown nonthermal power law spectra from the coronal sources and thus resolved the above controversy (e.g. Krucker et al. 2008a, 2010).

The aforementioned LT and FP sources are associated with a population of electrons propagating downward along a closed loop below the current sheet and constitute the majority of the flare HXR emission. The bipolar X-type reconnection model also suggests existence of another electron beam above the current sheet, which may propagates upward along open field lines through the corona (e.g. Sturrock 1966; Aschwanden 2002). A second coronal HXR source, which appears to be located above the LT coronal source, has been detected recently by RHESSI up to $\sim 20-30 \mathrm{keV}$ in a few events (e.g. Sui \& Holman 2003; Sui et al. 2004; Veronig et al. 2006; Li \& Gan 2007; Liu et al. 2008). These two coronal sources exhibit an opposite spatial gradient, for which the lower (upper) source appears at a higher (lower) altitude with increasing energy, indicating a current sheet formed in between. Such observations provide further evidence for magnetic reconnection and particle acceleration in solar flares.

In this paper, we present $R H E S S I$ imaging and spectroscopic observation of very energetic coronal HXR sources up to 100-150 keV simultaneously with two FP sources in a solar flare on 2003 November 3 (Solar Object Locator: SOL2003-11-03T09:43). This is one of a few flares in which we found simultaneous LT and FP sources above $50 \mathrm{keV}$ observed by RHESSI during the impulsive phase (Chen \& Petrosian 2009). In Section 2 we present the imaging results of the coronal HXR sources. In Section 3 we mainly study the evolution of imaging spectroscopy of the LT coronal source and its comparison with the FP sources. In Section 4 we discuss the implications arising from the above results and a possible connection between the coronal HXR sources and the type III radio bursts observed during the impulsive phase of the flare. Finally, in Section 5 we briefly summarize our results.

\section{HIGH ENERGY CORONAL SOURCES}

The 2003 November 3 solar flare under study is an intense eruptive event occurring in NOAA Active Region

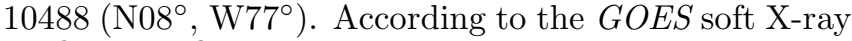
profiles, the flare starts at 09:43 UT, peaks at 09:55 UT, and ends at 10:19 UT, and is classified as an X3.9 event. This flare is accompanied with type III radio bursts and a coronal mass ejection event (Dauphin et al. 2005), but no solar energetic particles. Earlier analysis of the flare identified a pair of coronal sources below $\sim 30 \mathrm{keV}$ and two conjugate FPs based on the RHESSI HXR images reconstructed with the Clean method (Liu et al. 2004; Liu 2006; Veronig et al. 2006). We show below that the two coronal sources actually extend to much higher energies by means of different image reconstruction methods. These high energy coronal sources yield significantly new information about the coronal radiation regions.

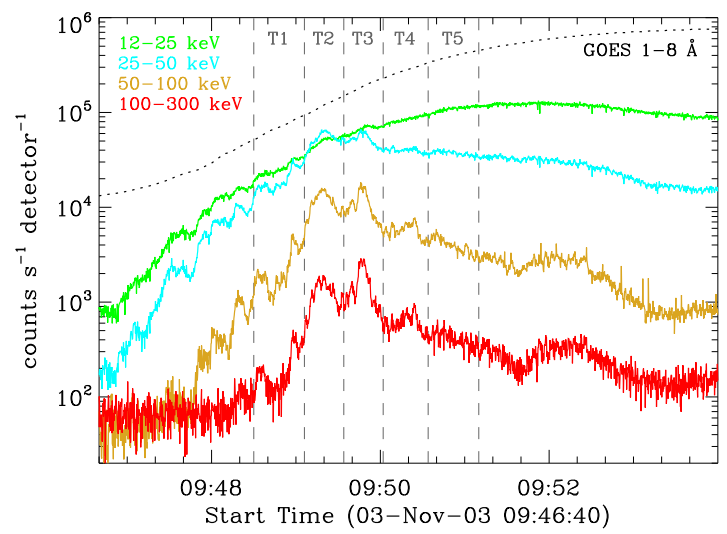

FIG. 1.- Demodulated RHESSI count rates with 0.25 s resolution at four broad energy bins from 12 to $300 \mathrm{keV}$, superposed with the GOES soft X-ray flux at 1-8 $\AA$ (dotted, in arbitrary units). The vertical lines (dash) delimit five time intervals for imaging and spectroscopic analysis, the third of which is the nonthermal peak. 

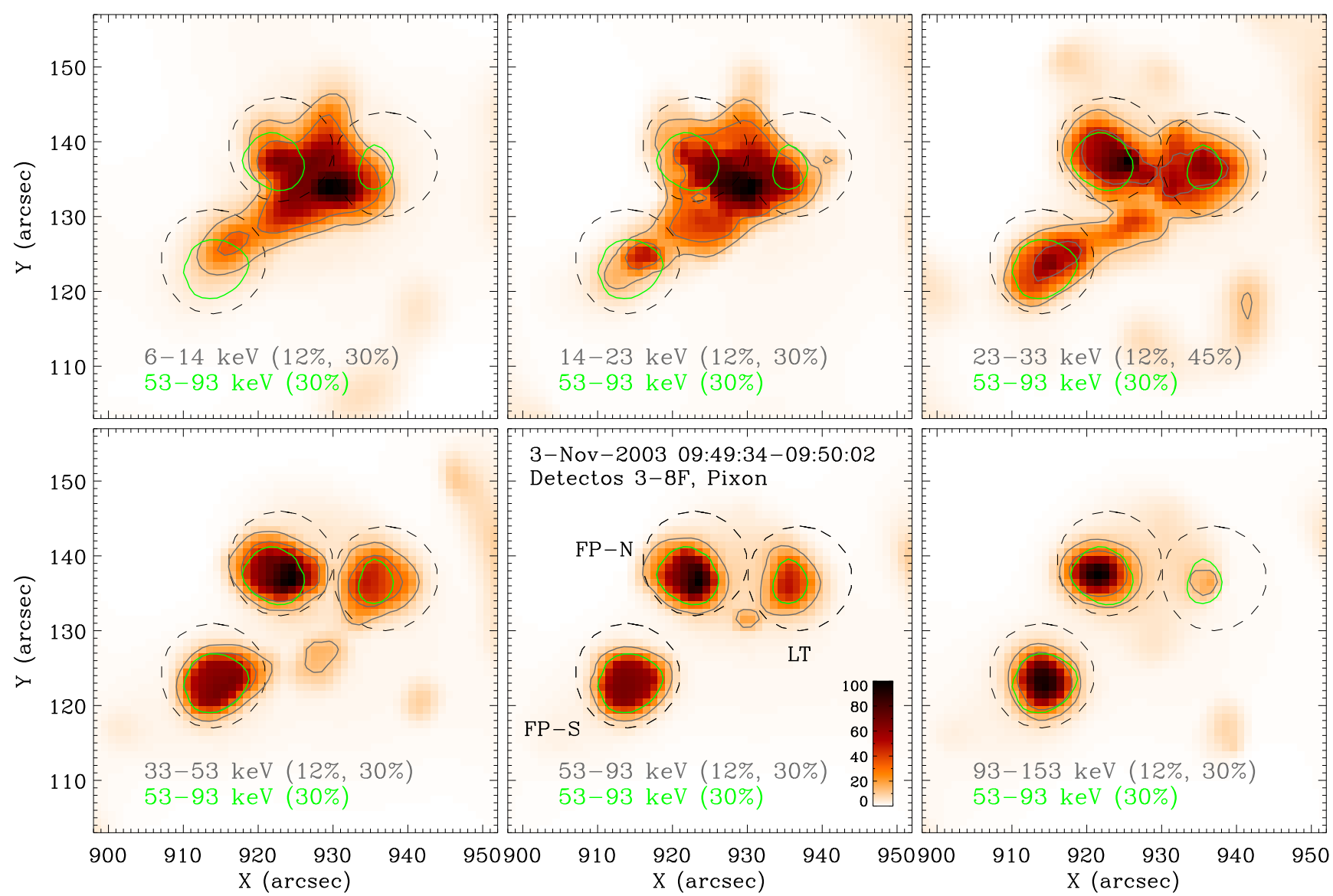

FIG. 2.- HXR images at six broad energy bins from 6 to $153 \mathrm{keV}$ during the nonthermal peak generated by Pixon from the front segments 3-8 of FWHM $\sim 6.8^{\prime \prime}$. Also shown are two contour levels of each image (gray) and the $30 \%$ level of the $53-93 \mathrm{keV}$ image (green). The four intermediate energy bins from 14 to $93 \mathrm{keV}$ are designed to resemble the Yohkoh/HXT energy bands. The images exhibit a loop structure below $23 \mathrm{keV}$, and two FP sources and one LT coronal source dominating at higher energies. The LT source at 33-153 keV falls within the $\sim 12 \%$ level of the thermal loop emission. Three circles (dash) with identical radii of $7^{\prime \prime}$ mark the regions from which HXR fluxes are extracted for spectral analysis. All images are scaled to the same color bar (bottom middle, in arbitrary units) for display.

We focus on the impulsive phase of the flare (see Figure 1). The RHESSI count rates above $25 \mathrm{keV}$ show two main peaks around 09:49:20 and 09:49:48 UT, while the rates at lower energies increase nearly monotonically. In Figure2 2 we show the HXR images and source contours at six broad energy bins $(6-14,14-23,23-33,33-53,53-93$, and $93-153 \mathrm{keV}$ ) during the nonthermal peak as generated by the Pixon algorithm from the front segments 3-8. The images from 6-23 keV show an asymmetric cuspshaped loop structure and faint, yet discernible emission from the southern FP of the loop. At higher energies up to $93-153 \mathrm{keV}$, two FPs and one LT source dominate the HXR emission. The $\geq 30 \mathrm{keV}$ component of the LT source is clearly revealed by the MEM_NJIT algorithm and the Clean components as well, but was missed in earlier analysis of the flare based on the Clean images. We include comparison of these different algorithms in Appendix A. We also discuss the pulse pileup effect in Appendix $\mathrm{B}$ and conclude that the LT source should not be due to the pileup effect.

This high energy LT source occurs nearly throughout the 2003 November 3 solar flare and is most prominent during the impulsive phase. Its maximum intensity is about $\sim 50 \%$ of the maximum FP intensity at $33-93 \mathrm{keV}$ and $\sim 20 \%$ at $93-153 \mathrm{keV}$. As shown in Section 3 , the LT flux at $50 \mathrm{keV}$ is $\sim 4.5$ photos $\mathrm{s}^{-1} \mathrm{~cm}^{-2} \mathrm{keV}^{-1}$, compa-

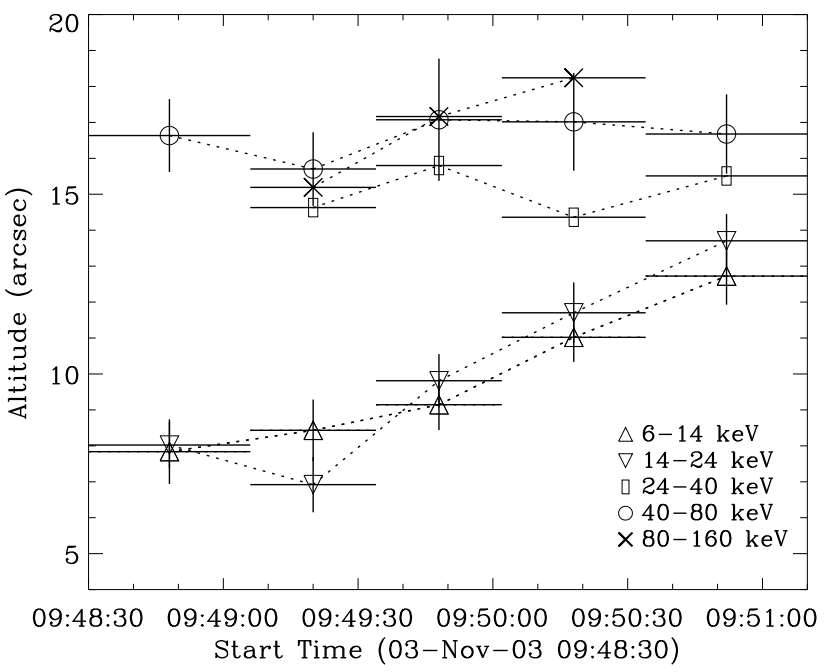

FIG. 3.- Altitude of the LT centroid from 6-160 keV as a function of time. Also shown are the error bars for the 6-14, 14-24, and 40-80 keV energy bins. The uncertainty of the source centroid is estimated following Bogachev et al. (2005) and Mrozek (2006), which for the current flare is approximated by the FWHM divided by the square root of the number of pixels within the $50 \%$ contour level.

rable to the typical FP flux of an X1 class flare (see Figure 9 of Saint-Hilaire et al. 2008). Furthermore, the dis- 

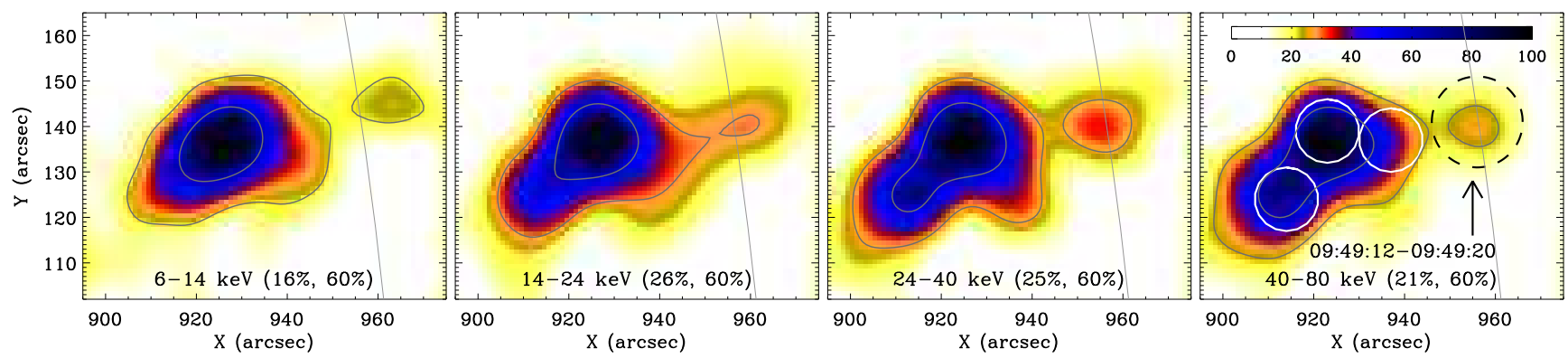

FIG. 4.- Upper coronal source seen at four energy bins from 6 to $80 \mathrm{keV}$ during part of the first peak (09:49:12-09:49:20 UT), as indicated by the upward arrow and a circle of radius $10^{\prime \prime}$ (dash) in the rightmost panel. The images are generated by the Clean algorithm (with natural weighting) from the front segments $3-8$, superposed with two contour levels of each image. The color bar is customized to highlight the upper coronal source. The three smaller circles mark the LT and two FP sources seen in the Pixon images (see Figure 2). The solid curve denotes the solar limb.

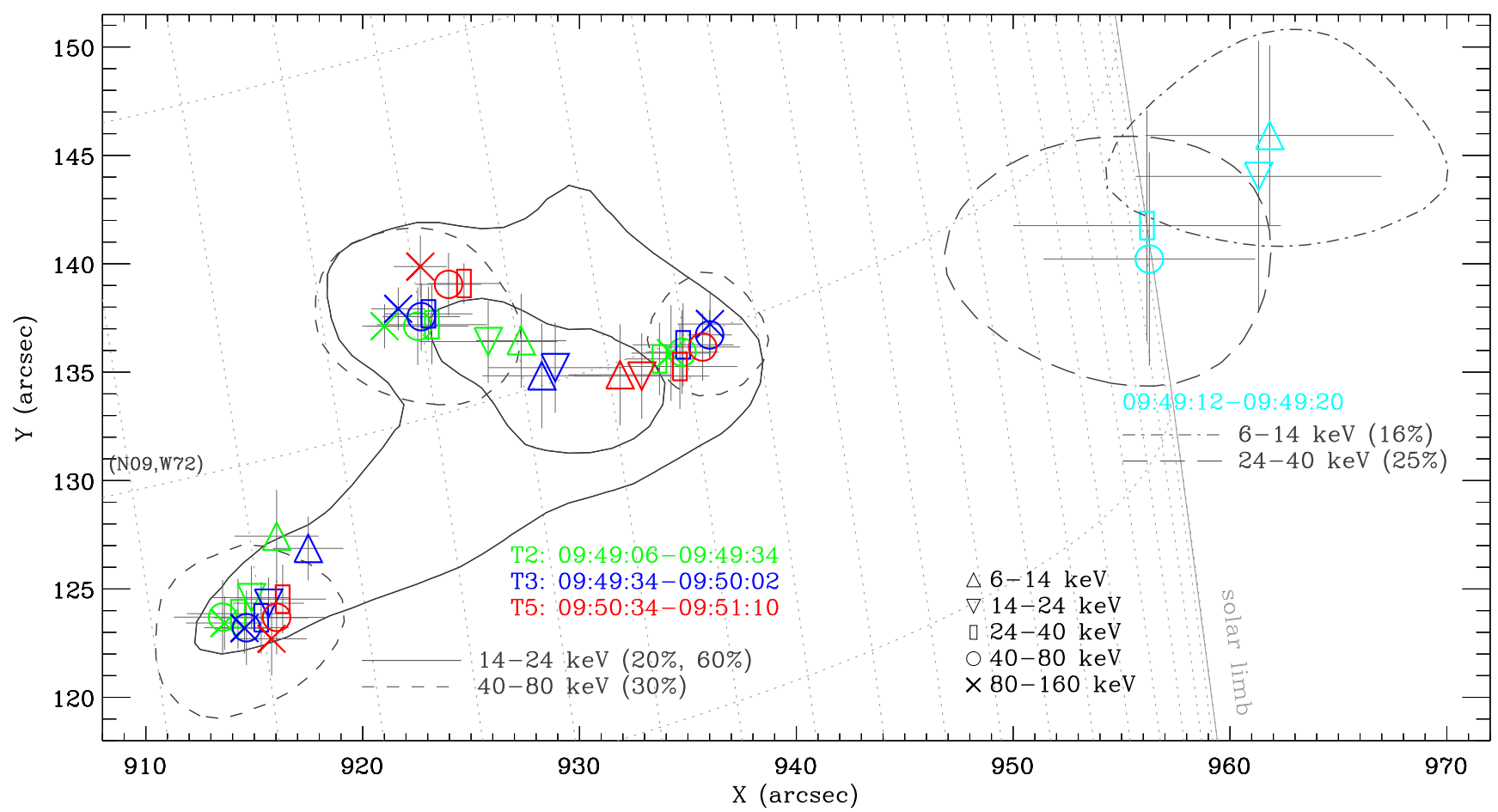

FIG. 5.- Centroid distribution of individual HXR sources during the impulsive phase. The left part shows the centroids of the LT and FP sources from the Pixon images at five broad energy bins from 6-160 keV in three time intervals, superposed on the 14-24 keV (solid) and $40-80 \mathrm{keV}$ (dash) contours from the nonthermal peak. The LT centroids are mainly distributed along the circle of latitude at N09 ${ }^{\circ}$. The right part shows the centroids of the upper coronal source from the Clean images (with natural weighting) during part of the first peak, supposed on the 6-14 keV (dash dot) and 24-40 keV (long dash) contours. The centroids are calculated as the source position weighted with the intensity within the $\sim 50 \%$ contour level of each source. The horizontal and vertical bars show the standard deviations of the centroids. The dotted lines show the heliographic grids with $1^{\circ}$ separation.

tinct LT source above $23 \mathrm{keV}$ clearly lies above the most intense part of the thermal loop and is well separated from the FP sources. The projected angular separation between the centroids of the high energy LT and the thermal loop is $\sim 8^{\prime \prime}$. This unusual LT source observed by RHESSI is reminiscent of the "above-the-loop-top" coronal source seen up to $33-53 \mathrm{keV}$ in the 1991 January 13 flare observed by Yohkoh/HXT, which lies above the thermal soft X-ray loop by $\sim 10^{\prime \prime}$ (Masuda et al. 1994). In comparison, the nonthermal and thermal components of the coronal sources are mostly cospatial as observed in partially occulted flares (Tomczak 2001, 2009; Krucker \& Lin 2008c). On the other hand, despite its large separation from the intense thermal loop, this high energy coronal source should still be located at the top of some cusp-shaped magnetic loop, which may not yet become fully visible at soft X-rays (see also Liu et al. 2008; Longcope \& Guidoni 2011). Therefore, in this paper, we use the term "loop top (LT)" for this coronal source.

Earlier analysis of the flare showed that the thermal LT moves upward (after an early altitude decrease) and the two FPs move apart as the flare proceeds in time (Liu et al. 2004; Veronig et al. 2006), supporting the standard magnetic reconnection model (e.g. Priest \& Forbes 2000). Now we investigate the spatial distribution of the LT source at different energies. In Figure 3 we plot the altitude of the centroids of the LT source within its $\sim 50 \%$ contour level at five broad energy bins (6-14, 14-24, 24-40, 40-80, and 80-160 keV) in five time intervals during the impulsive phase (see Fig- 
ure 1). First, in general the centroids appear at higher altitudes with increasing energy (see also Figure 5) and the centroids above $24 \mathrm{keV}$ show a displacement of $\leq 8^{\prime \prime}$ from those at lower energies. Second, the LT centroids below $24 \mathrm{keV}$ move gradually to higher altitudes (up to $\sim 6^{\prime \prime}$ ) with a velocity $\sim 30 \mathrm{~km} \mathrm{~s}^{-1}$ as the flare develops. While at higher energies, the LT source shows little motion except at $80-160 \mathrm{keV}$. These behaviors indicate very efficient confinement of the accelerated electrons in the solar corona.

Furthermore, we show in Figure 4 that the second (upper) coronal source sitting around the west solar limb (Veronig et al. 2006) actually has significant emission even at energies up to $\sim 80 \mathrm{keV}$. This high coronal source can be best detected in the Clean images around the first peak of the flare and with a relatively short integration time. It appears rather distinctive and separated from the underlying closed loop consisting of the LT and FP sources. Its altitude is roughly 2-3 times that of the high energy LT source. We plot in Figure 5 the spatial distribution of its centroids, along with the LT and FP centroids. The energy dependence of its centroids exhibits nearly an opposite trend compared to the LT; the higher energy this coronal source, the lower its altitude toward the flare loop. In other words, the centroids of both coronal sources are closer to the imagined reconnecting X-point at higher energies. This flare provides an example of a current sheet (of an extent $\sim 20^{\prime \prime}$ ) as inferred from X-ray observation of the outflow regions with much higher energies than other events.

\section{IMAGING SPECTROSCOPY}

In this section we present results from imaging spectroscopic analysis of individual HXR sources, which is implemented using the Object Spectral Executive (OSPEX; Smith et al. 2002) package of the Solar SoftWare (SSW). We use the Pixon algorithm to reconstruct images from 6 to $\sim 180 \mathrm{keV}$. We then extract the spatially resolved HXR spectra (photons $\mathrm{cm}^{-2} \mathrm{~s}^{-1} \mathrm{keV}^{-1}$ ) from the LT and FP sources over three fixed circles with radii $7^{\prime \prime}$ (see Figure 21). Following the procedure currently adopted in OSPEX for error estimate (see e.g. Saint-Hilaire et al. 2008), we take one third of the maximum flux outside the flaring region to be the 1- $\sigma$ uncertainty of the flux of each source. Finally we fit the HXR spectra parametrically to the combination of an isothermal bremsstrahlung spectrum and a single or broken power law (Holman et al. 2003) using RHESST's full spectral response matrix (e.g. Liu et al. 2008).

\subsection{HXR Spectra}

In Figure 6, we show the HXR spectra $I(\epsilon)$ for the LT and the summed FPs and the corresponding fitting. The LT spectra can be well fitted by a thermal function plus a power law tail with an index $\gamma \equiv-d \ln I(\epsilon) / d \ln \epsilon$ varying from $\sim 4-5.5$. If we take the emission measure $\mathrm{EM}=0.3 \times 10^{49} \mathrm{~cm}^{-3}$ and the size $L=10^{9} \mathrm{~cm}$, and assume a filling factor of unity, we obtain a density $n=\sqrt{\mathrm{EM} / L^{3}} \simeq 5 \times 10^{10} \mathrm{~cm}^{-3}$ averaged over the circle "LT" in Figure 2. Here we should note that since the circle "LT" includes part of the low energy thermal loop and the neighboring pixels reconstructed from indirect Fourier imaging (as employed by RHESSI) are not independent, the thermal emission within this circle mainly comes from part of the thermal loop. Therefore the thermal properties of the high energy LT source cannot be well measured and the above value $n$ may only give an upper limit for the LT density, which could be significantly lower. In comparison, the average density of the entire thermal loop is estimated to be $\sim 8 \times 10^{10} \mathrm{~cm}^{-3}$.

In Figure 6 top right panel, we also plot the spectrum of the upper coronal source that is located above the LT source during the first peak of the flare. It turns out that the two coronal sources have comparable spectral indices. The similarity in HXR spectra may provide further evidence that the same acceleration mechanism is responsible for the two oppositely directed electron beams that generate the two coronal HXR sources.

In contrast, the FP spectra above $\sim 20 \mathrm{keV}$ are stronger and flatter and are better fitted by a broken power law. At lower energies, the FP spectra show a softer, perhaps quasi-thermal component which can be fitted by a thermal function of a temperature $\sim 2 \times 10^{7} \mathrm{~K}$. In particular, the southern FP source, which has little contamination from the intense loop emission (see Figure 21), clearly shows such a prominent thermal-like component. Thermal X-ray emission from the FP regions has been detected from a few other flares (e.g. McTiernan et al. 1993; Hudson et al. 1994; Battaglia \& Benz 2006) with a temperature as high as $\sim 10^{7} \mathrm{~K}$ (Hudson et al. 1994). The origin of the thermal-like component of the FP spectra in the current flare is puzzling (see also Section 4.1).

\subsection{Spectral Relation and Evolution}

Now we investigate the temporal evolution of the LT and FP spectra and their relative difference as shown in Figure 7. First, in the top panel, we show the time profiles of the fluxes from the LT and the summed FP sources with a time resolution of 4 seconds. Below 25 $\mathrm{keV}$, both the LT and FP fluxes show gradual increase from the beginning; while above $25 \mathrm{keV}$, they show impulsive profiles that are well correlated but there is also some difference. Also note that the ratio between the LT and the FP fluxes increases with time (i.e. the LT becomes relatively more prominent compared to the FPs). This could be partly due to the successive formation of larger coronal loops as reconnection proceeds and the effect of chromospheric evaporation, which fills the coronal loop with dense chromospheric plasma, making bremsstrahlung emission brighter around the LT region.

Second, as in the middle panel, the spatially resolved LT and FP spectra roughly exhibit soft-hard-soft evolution, namely, the spectral index is anti-correlated with the flux during the flare. Note that for the FP spectra, we take the average value of the two indices below and above the break energy. We have also fitted the LT and FP spectra above $50 \mathrm{keV}$ by a power law, as similarly done by Emslie et al. (2003) and Liu et al. (2009a), and found a similar trend as above. This behavior has been found in the spatially integrated spectra (e.g. Grigis \& Benz 2004) or spatially resolved spectra from RHESSI (e.g. Battaglia \& Benz 2006). Such spectral evolution may reflect an intrinsic property of the acceleration mechanism and can be explained by the stochastic acceleration model (e.g. Petrosian \& Liu 2004; Grigis \& Benz 2006; Bykov \& Fleishman 2009; Liu \& Fletcher 2009). However, there is no exact peak to peak correspondence seen in this flare. 


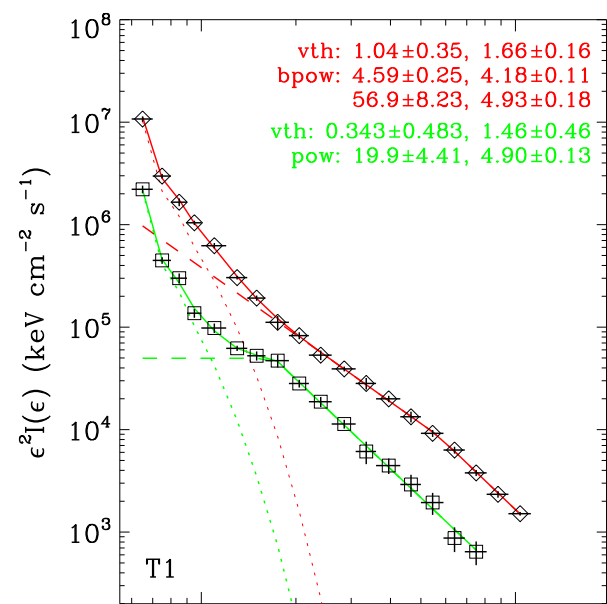

10

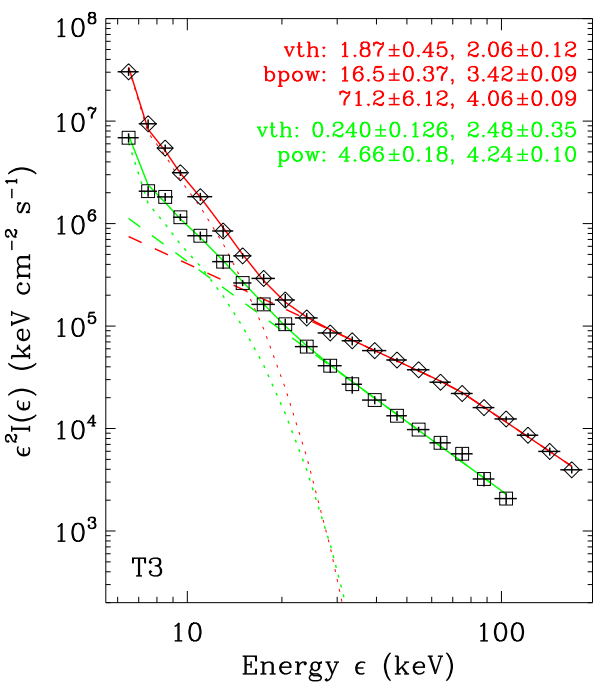

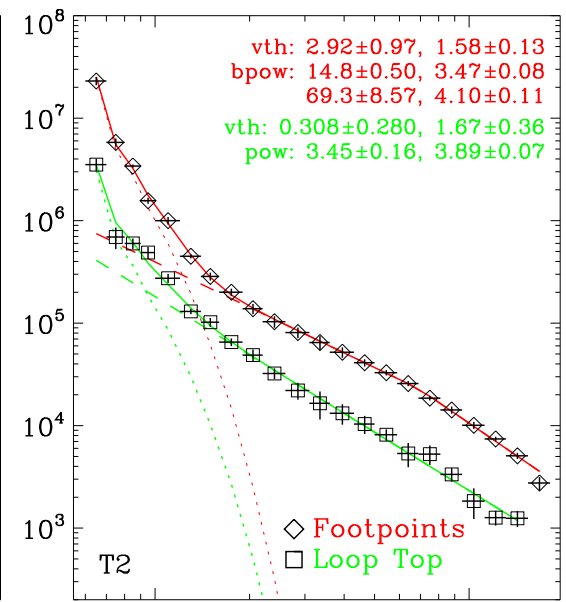

10

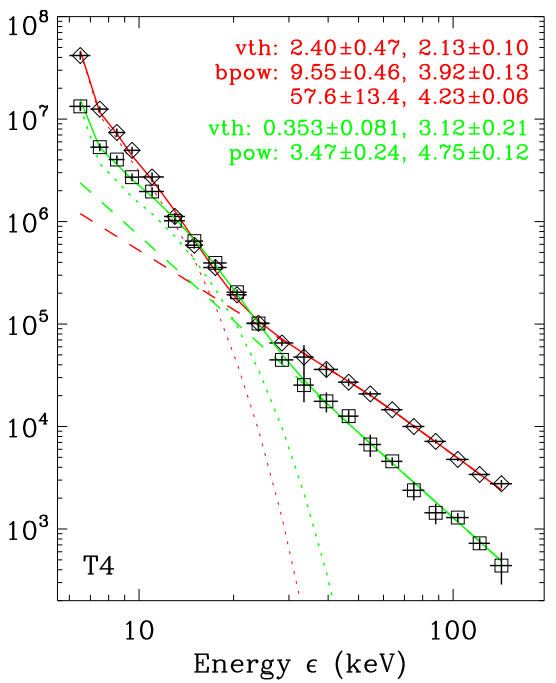

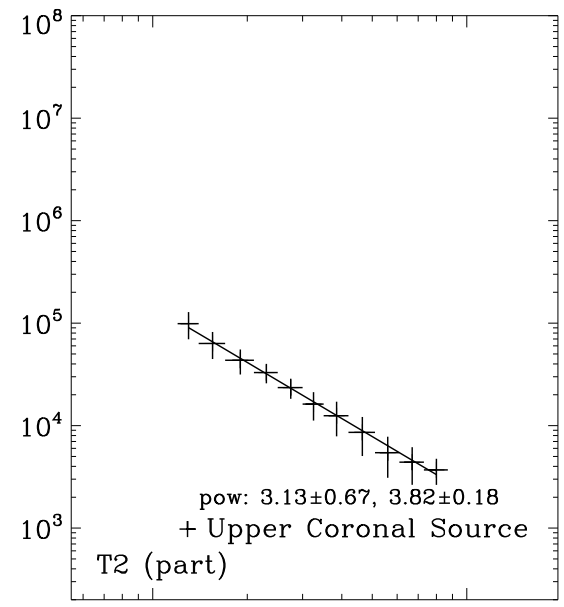

10

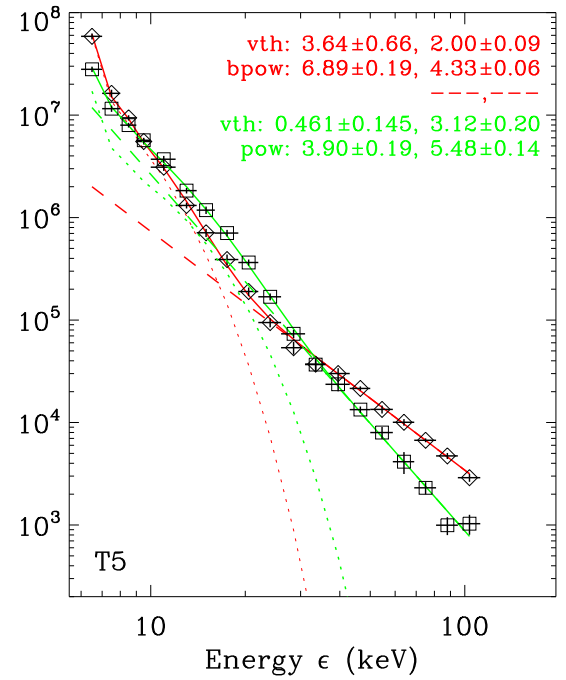

FIG. 6.- HXR spectra $I(\epsilon)$ from the LT source (square, green) and the two FP sources summed (diamond, red) in five time intervals (see T1-T5 in Figure 1). The spectra are fitted by an isothermal bremsstrahlung spectrum plus a single or broken power law from 6 keV to the highest energies at which the sources are visible. For the first interval, we also include a low energy break at $\sim 20 \mathrm{keV}$. As for the fitting parameters, the isothermal function ("vth") includes the emission measure (EM, in units of $10^{49} \mathrm{~cm}^{-3}$ ) and temperature (T, keV); the single power law ("pow") includes the normalization of the spectra at $50 \mathrm{keV}$ and the spectral index $\gamma$; the broken power law ("bpow") further includes the break energy and the index above the break. Note that below $\sim 20 \mathrm{keV}$, the LT spectra have significant contamination from the thermal loop emission and thus the displayed thermal parameters do not faithfully represent the LT source; also the northern FP source partly overlaps the loop emission, thus the summed FP flux is overestimated. The top right panel shows the spectrum from the upper coronal source (plus) during 09:49:12-09:48:24 UT and the corresponding power law fitting. In order to highlight the difference between the steep LT and FP spectra, we have multiplied $I(\epsilon)$ with $\epsilon^{2}$ for display. This representation also indicates the photon energy at which most of the energy is radiated.

Third, as in the bottom panel, the difference between the LT and the FP spectral indices, $\Delta \gamma=\gamma_{\mathrm{LT}}-$ $\gamma_{\mathrm{FP}}$, is very small during the impulsive phase. It is smaller than or around one, being around zero during the first peak. We have also fitted the ratio between the LT and FP spectra by a power law and found a similar index difference. The difference here is comparable to or even smaller than the most extreme cases found in previous statistical study for the Yohkoh flares (Metcalf \& Alexander 1999; Petrosian et al. 2002) and RHESSI flares (Battaglia \& Benz 2006; Liu 2006; Shao \& Huang 2009). It is also considerably smaller than the average spectral difference between the (LT) coronal emission from partially occulted flares and the FP emission from non-occulted flares, $\sim 1.5$ for the Yohkoh flares (Tomczak 2009) and $\sim 2$ for RHESSI flares (Krucker et al. 2008a; Krucker \& Lin 2008c). The ob- served small spectral difference reflects why the 2003 November 3 flare has very bright LT emission up to $\sim 100-150 \mathrm{keV}$ that can still be detected simultaneously with the intense FPs under the current dynamic range $\sim 10$.

\section{DISCUSSIONS}

The imaging and spectroscopic analysis of the coronal HXR sources in the 2003 November 3 flare observed by RHESSI strongly indicate that electron acceleration is closely related to the energy release process through the reconnecting current sheet in solar flares. The spatial variation of the LT coronal source may be indicative of the cusp-shaped geometry of the outflow region below the current sheet. Detection of coronal sources extending to high energies can severely challenge theoretical models and constrain intrinsically complex physical processes in solar flares. In this section, we first discuss a few aspects 

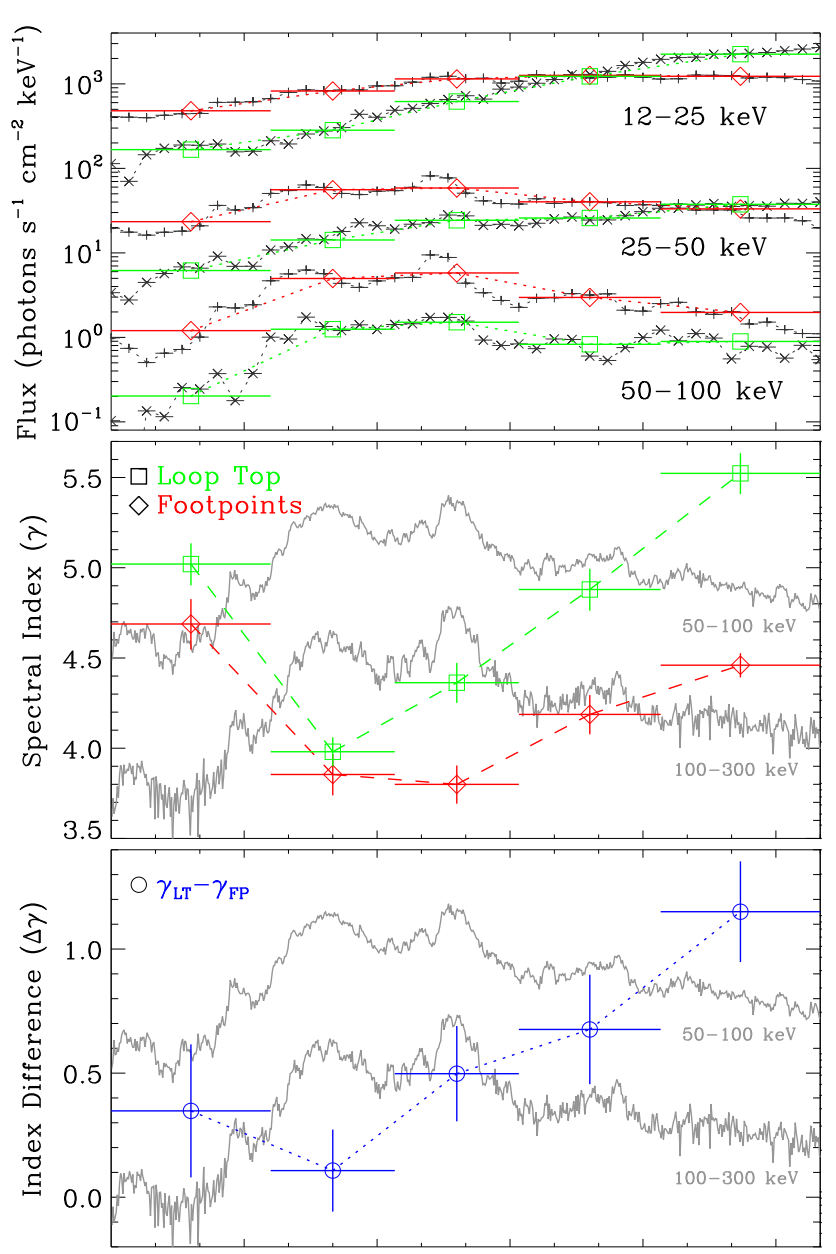

09:49:00 09:49:30 09:50:00 09:50:30 09:51:00 Start Time (03-Nov-03 09:48:30)

FIG. 7.- Spectral evolution during the impulsive phase. Top: HXR fluxes from the LT source (square, green) and the two FP sources summed (diamond, red) at $12-25,25-50$, and $50-100 \mathrm{keV}$. Also shown are the $4 \mathrm{~s}$ resolution data (black). The fluxes are extracted from the Pixon images. Middle: (mean) power law indices of the LT and the summed FP spectra. Bottom: difference in power law indices (circle, blue) between the LT and the FPs. The lower two panels are superposed on the spatially integrated fluxes at 50-100 and $100-300 \mathrm{keV}$ (in arbitrary units).

mainly arising from the newly detected high energy LT coronal source and compare them with some previous models, in particular, the stochastic acceleration model. Second, we attempt to explore a possible connection between the coronal HXR sources and the type III radio bursts observed during the impulsive phase of the flare.

\subsection{Spatial Structure}

Existence of the distinct coronal sources up to $\sim 100$ $150 \mathrm{keV}$ clearly implies that the electrons are accelerated to $\geq 200-300 \mathrm{keV}$ and are confined near these sources. This may result from pitch angle scattering of electrons by turbulence, as advocated by the stochastic acceleration model (e.g. Petrosian \& Donaghy 1999; Petrosian \& Liu 2004). The opposite spatial gradient of two coronal sources observed in this and a few other events, in which the sources get harder toward the presumed reconnection X-point, can also be explained by this model (Liu et al. 2008). In addition, the electron escape time from the acceleration site in this flare in- creases with energy (Petrosian \& Chen 2010), effectively enhancing their confinement (see, however, the discussion below). In this and other models the electrons escaping from the LT coronal region will produce HXR emission at the thick target FP regions and drive evaporation flow to fill the coronal loop, while those electrons escaping from the upper coronal source may be responsible for the type III radio emission (see Section 4.4 below).

Another model often advocated for trapping of electrons in the corona is magnetic field convergence below the current sheet (Fletcher \& Martens 1998). Such a magnetic bottle can generate a distinct LT coronal source, but not the variation of the source centroid with energy. Recently, a drift-kinetic model including betatron acceleration and collisional pitch angle scattering during field line shrinkage (Minoshima et al.|2011) shows that the height of the accelerated electrons in the corona increases with energy up to a few tens of $\mathrm{keV}$, but then decreases at higher energies. This may qualitatively explain previous observations of the height distribution of low energy coronal sources, but it cannot account for the increase of the coronal LT height at higher energies up to $\sim 100-150 \mathrm{keV}$ (with corresponding electron energy $\geq 200-300 \mathrm{keV}$ ) as observed in the current flare.

Ever since the observation of the above-the-loop-top source in the 1991 January 13 flare (Masuda et al. 1994), it is often stated that electron acceleration takes place above the thermal soft X-ray loop. However, the spatial variations described above indicate that the situation is more complex. Because the more abundant lower energy electrons are located at lower (or inner) field lines and because they are the more effective agent of heating and evaporation compared to the high energy electrons, it may be natural to expect the thermal loop to lie somewhat below the LT coronal HXR source. More thorough modeling including transport of electrons in an inhomogeneous environment is required to address these details.

Finally, we briefly discuss the spatial distribution of the FP sources. They in general appear deeper at the lower part of the loop with increasing energy (see Figures 2 \& 5), which at first sight seems consistent with the classical thick target model for electron transport. However, the existence of the low energy southern FP source at $\sim 6 \mathrm{keV}$ is difficult to explain when considering that the column density along the loop leg $\sim 8 \times 10^{19} \mathrm{~cm}^{-2}$ (see Section 3) can collisionally stop electrons with energy 1 up to 20 $\mathrm{keV}$. Although quasi-thermal electrons may escape from the corona as shown in the stochastic acceleration model (Petrosian \& Liu 2004), the above collisional effect basically leads to little low energy thermal emission at the FPs (e.g. Figure 12 of Liu et al. 2009b). In addition, the gradual rising time profiles of the FP emission at such low energies do not seem to agree with the scenario of direct heating due to nonthermal electrons at the FP regions (Hudson et al. 1994). Therefore the origin of the low energy FP sources of quasi-thermal spectra observed in this flare is not clear. It deserves further study in the future whether the current observation requires more valid transport models for energetic electrons escaping from

${ }^{1}$ In the classical thick target model (e.g. Brown et al. 2002), a column density $\mathcal{N}$ can stop (nonrelativistic) electrons with energies up to $E=7.2\left(\mathcal{N} / 10^{19} \mathrm{~cm}^{-2}\right)^{1 / 2} \mathrm{keV}$, if the Coulomb logarithm $\ln \Lambda=20$. 
the corona or implies some new energization processes in the dense chromosphere (e.g. Fletcher \& Hudson 2008; Brown et al. 2009).

\subsection{Spectral Properties}

The 2003 November 3 flare shows the usual soft-hardsoft evolution of the LT and FP spectra (Figure 7 , middle panel). This is a natural consequence of the stochastic acceleration model, where the spectral hardness of the accelerated electrons, and consequently that of the emitted HXR photons increase primarily with the level of turbulence intensity (e.g. Petrosian \& Liu 2004). A higher level of turbulence may also cause more efficient trapping, which is equivalent to slower escaping of the accelerated electrons to the FPs of the loop. This may be the explanation for the evolution of the LT and FP spectral difference (Figure 7 , bottom panel).

However, the most unusual spectral aspect of this flare is the observation of the LT source up to high energies $(\sim 100-150 \mathrm{keV})$, which is made possible because of its relatively hard spectrum. As emphasized above, the observed spectral index difference between the LT and FP sources $(\Delta \gamma \simeq 0-1)$ is significantly smaller than those found in the more frequent, less intense flares. Quantitatively, this spectral difference is determined by the energy dependence of the escape time in the acceleration region, which is related to the pitch angle scattering rate. As derived directly from the electron flux images, the escape time from the LT acceleration site during the nonthermal peak of the flare increases with energy (Petrosian \& Chen 2010), requiring a scattering time that is shorter than the crossing time and decreases with energy relatively rapidly. This does not seem to have a simple explanation considering the models proposed so far for the LT coronal emission.

First, we note that in previous literature for stochastic acceleration (e.g. Miller et al. 1990; Prvadko \& Petrosian 1997, 1998; Chandran 2003; Emslie et al.|2004; Petrosian \& Liu 2004; Grigis \& Benz 2006; Bykov \& Fleishman 2009; Liu \& Fletcher 2009), the electron escape time is either simply taken to be the same as the crossing time $\tau_{\text {cross }}=L / v \propto 1 / \sqrt{E}$, which is the case when the scattering time $\tau_{\text {scat }} \gg \tau_{\text {cross }}$, or set to $\tau_{\text {cross }}^{2} / \tau_{\text {scat }}$ for the opposite case. The scattering time can be calculated numerically assuming a Kolmogorov-type spectrum of turbulence and/or parallel propagating plasma waves along magnetic field lines. The latter also gives rise to an escape time that is very flat or decreases with electron energy (e.g. Petrosian \& Liu 2004). The escape time of such an energy dependence roughly leads to $\Delta \gamma \simeq 1.5-2$ between the LT and FP spectra in the energy range of interest for HXR observations.

One of the earliest models suggested for production of a distinct LT source is collisional confinement by a dense region in the corona (Wheatland \& Melrose 1995). In this model, the high energy LT source is thin target in nature and its spectrum is softer than the thick target $\mathrm{FP}$ spectrum by $\Delta \gamma \simeq 2$. One may include transport effects from the corona to the chromosphere, such as return current energy loss (e.g. Zharkova \& Gordovskvy 2006; Battaglia \& Benz 2008), a nonuniform ionization target (e.g. Su et al. 2009), and wave-particle interactions (e.g. Holman et al. 1982; Hannah \& Kontar 2011), to overcome the difficulty. However, all these effects in general tend to make the electron spectrum flatter and thus cause $\Delta \gamma>2$.

One model based on collisional pitch angle scattering of electrons is that of converging magnetic field lines in the corona (e.g. Fletcher \& Martens 1998). This may effectively increase the electron escape time with energy to be $\propto E^{3 / 2}$ (see Melrose \& Brown 1976). However, as shown in Petrosian \& Chen (2010), the required escape time varies less rapidly with energy than $E^{3 / 2}$, and more importantly, it is about ten times smaller than the collisional energy loss time for this flare. In addition, this effect alone tends to make the coronal LT spectrum progressively harder with time and the LT flux decay slower than the FP flux, which contradict what are observed during the impulsive phase (see Figure 7).

Therefore, it seems that in this special flare one needs to more effectively confine the high energy electrons in the acceleration region than predicted by the above models. We believe that the observed small spectral difference during the impulsive phase should most likely be related to some unique conditions or the acceleration or transport processes in the corona. For example, as pointed out in Petrosian \& Chen (2010), one possibility to increase the escape time for high energy electrons is to invoke a turbulence spectrum that is steeper than the commonly assumed Kolmogorov-type spectrum, as is expected for damped turbulence beyond the inertial range. Another possibility is acceleration by perpendicularly propagating plasma waves, which can preferentially accelerate electrons with the pitch angle near $90^{\circ}$ (e.g. Petrosian \& Donaghy 1999). Compared to the unidirectional beam distribution or isotropic distribution, such a pancake-like distribution can more easily confine electrons in the corona. Finally, it is likely that rather than operating separately, some of these conditions may be operating simultaneously and be capable to account for the observed spectral difference and its evolution. A combination of turbulence scattering and magnetic field convergence may possibly yield an escape time increasing with energy, yet much shorter than the collisional loss time.

\subsection{Thermal and Nonthermal Electrons}

Conventionally, the thick target bremsstrahlung of nonthermal electrons injected into the loop is used to model the spatially-integrated HXR emission from the flare loop. While for the current flare under study, imaging spectroscopic observation of the spatially resolved LT and FP sources over a range $>100 \mathrm{keV}$ provides a unique opportunity to infer the processes of electron heating, acceleration, and escaping in the corona. Assuming that the LT source is resulting from the thin target bremsstrahlung and the FP sources are due to thick target emission by the escaping electrons, both with an addition of a thermal component, we adopt the forward fitting method (Holman et al. 2003) to independently infer the accelerated electron flux spectrum at the LT and the escaping (or injected) electron flux spectrum to the FP sources.

As shown in Figure 8 during the flare nonthermal peak, the electron flux spectrum at the LT can be well fitted by a power law with an index $\delta \simeq 3.3$ and a 


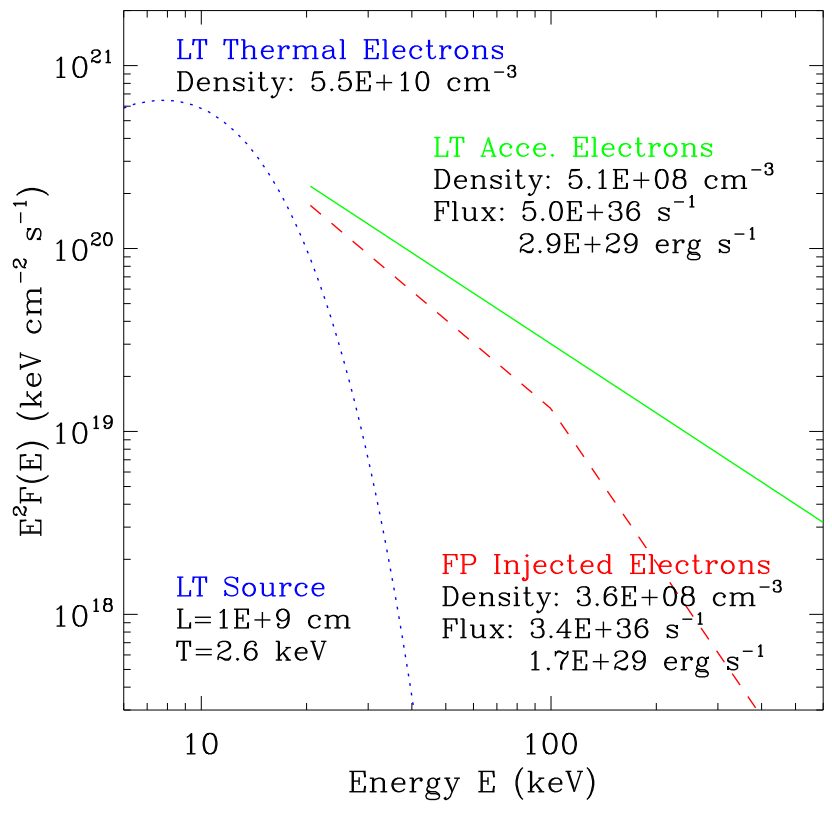

Fig. 8.- Flux spectra $F(E)$ of the accelerated electrons as inferred from the thin target LT bremsstrahlung spectrum (for a background density $\sim 5 \times 10^{10} \mathrm{~cm}^{-3}$ ) and the escaping (injected) electrons from the thick target FP spectrum during the nonthermal peak ( 28 seconds) by parametric forward fitting. The LT electron spectrum can be well fitted by a power law (solid, green) with $\delta \simeq 3.3$ and $E_{\mathrm{c}}=20 \mathrm{keV}$. The FP electron spectrum can be better fitted by a broken power law (dash, red) with the break energy set at $100 \mathrm{keV}$ and an index $\sim 3.6$ and $\sim 4.8$ below and above the break, respectively. Also shown is the thermal electron distribution at the LT source (dotted, blue). Note that the prescribed two-component model in the forward fitting procedure does not guarantee a continuous transition of the electron spectra.

low energy cutoff $E_{\mathrm{c}}=20 \mathrm{keV}$, and the (instantaneous) nonthermal electron density is $\sim 5 \times 10^{8} \mathrm{~cm}^{-3}$ assuming a background density $\sim 5 \times 10^{10} \mathrm{~cm}^{-3}$ (see Section 3). While the injected spectrum to the FPs can be better fitted by a broken power law, which is steeper and weaker than the LT accelerated spectrum. This difference may result from that the low energy electrons escape more easily from the corona to the FP regions than higher energy electrons and that the escape time is longer than the crossing time (Petrosian \& Chen 2010).

Given an upper limit of the background density $\sim 5 \times$ $10^{10} \mathrm{~cm}^{-3}$ and temperature $\sim 2.5 \mathrm{keV}$, the density and total energy of the accelerated electrons $\geq 20 \mathrm{keV}$ are roughly $1 \%$ and $10 \%$ (lower limits) of those of the background plasma, respectively. Such numbers are representative of the impulsive phase. It is worth mentioning that the above estimate of the number percentage of the accelerated electrons is inversely proportional to the background density squared or the emission measure at the nonthermal LT source.

On the contrary, if the LT density is indeed significantly lower than the above upper limit (see discussion in Section 31), then essentially all the background electrons would be accelerated into a power law. This will be similar to recent measurements of the above-theloop-top coronal sources observed by RHESSI in a C 8.3 class flare (Krucker et al. 2010) and an M9.9 class flare (Ishikawa et al. 2011b). Accordingly, the electron flux spectrum at the LT source (see Figure 8) will increase by $\sim 10$ times compared to the original assessment, so will

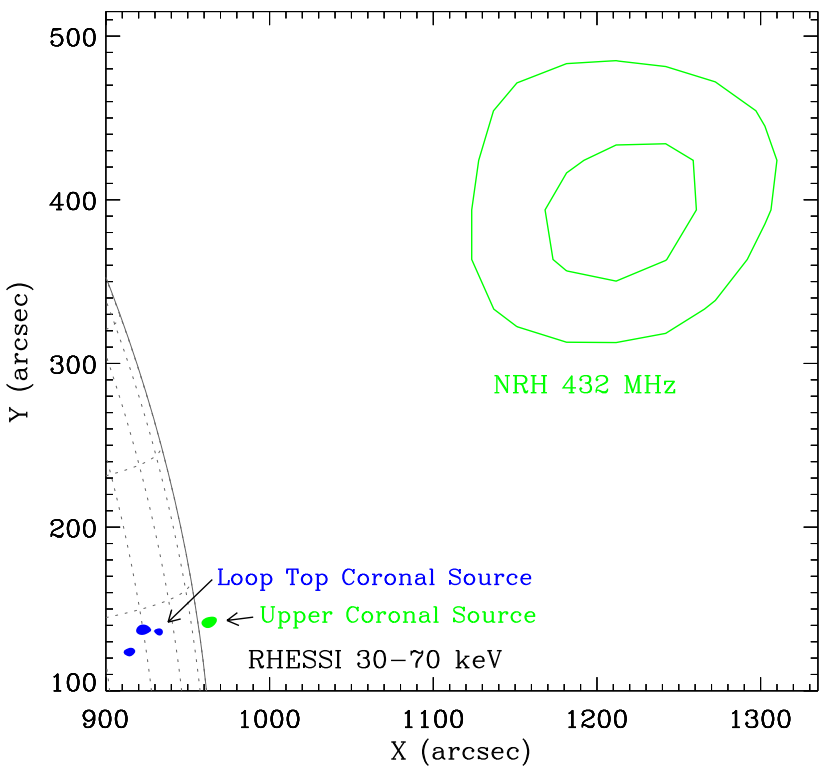

FIG. 9.- NRH $432 \mathrm{MHz}$ radio source (40\% and $80 \%$ contour levels, green) at 09:49:27 UT with an integration time of $0.9 \mathrm{~s}$ and the RHESSI HXR sources at 30-70 keV from 09:49:24-09:49:32 UT. Two arrows are drawn to point to the LT coronal source (blue) and the upper coronal source (green) indicating two electron populations that are spatially separated. The dotted lines show the heliographic grids with $5^{\circ}$ separation.

the density of the accelerated electrons, which becomes to be $\sim 5 \times 10^{9} \mathrm{~cm}^{-3}$.

\subsection{Type III Radio Bursts and Coronal Sources}

The flare accelerated electrons with access to open field lines eventually escape from the corona and produce type III radio bursts, which are characterized as features rapidly drifting from high to low frequencies in radio spectrograms (e.g. Bastian et al. 1998). The close temporal correlation between the type III bursts and the spatially integrated HXR emission observed in many flare events suggests that the corresponding electron beams may come from a common injection site or result from the same acceleration process in the corona (e.g. Kane 1981; Aschwanden et al. 1995). Recently a few solar eruption events with simultaneous observations of type III radio bursts and RHESSI HXR emission have been analyzed (e.g. Vilmer et al. 2002; Krucker et al. 2008d; Christe et al. 2008; Bain \& Fletcher 2009; Reid et al. 2011). However, so far the relation between the electron populations producing type III bursts and individual HXR sources still remains elusive.

Ground-based radio observation of the 2003 November 3 flare indicates that the impulsive phase is associated with many episodes of type III radio emission rapidly drifting from $\sim 400-500 \mathrm{MHz}$ to lower frequencies in the decimetric/metric range (see Dauphin et al. 2005). The radio sources that are related to the type III bursts exhibit some spatial dispersion with frequency (Dauphin et al. 2005, 2006). In Figure 9, we plot only the radio source at 09:49:27 UT from the highest frequency of $432 \mathrm{MHz}$ observed by the Nançay Radioheliograph (NRH; Kerdraon \& Delouis 1997). The local plasma density corresponding to the onset frequency of the type III bursts is estimated to be $\sim 6 \times 10^{8} \mathrm{~cm}^{-3}$, if the plasma emission is at the second harmonic. This density 
is about two orders of magnitude lower than that at the LT region (see Section 3). By adopting a coronal density model for conditions above active regions which uses 10 times of the Baumbach-Allen formula (e.g. Paesold et al. 2001), such a density corresponds to a height of $\sim 0.23$ $R_{\odot}$ above the solar surface, very close to the observed location of the $432 \mathrm{MHz}$ radio source.

Generation of type III radio bursts during the flare impulsive phase requires that the accelerated electrons have access to open field lines in the corona. In the bipolar model, open field lines can only exist above the reconnecting X-point (e.g. Sturrock 1966; Aschwanden \& Benz 1997; Aschwanden 2002). The flare accelerated electrons above the X-point that produce the upper coronal source eventually escape from the lower corona and induce the observed type III radio bursts (Figure 9). This flare provides a unique opportunity to connect the upper coronal HXR source and the type III radio bursts through the electron population located above the $\mathrm{X}$-point.

\section{SUMMARY}

Finally, we briefly summarize our results of the newly found high energy coronal HXR sources in the 2003 November 3 solar flare as observed by RHESSI.

1. The LT coronal source can be detected up to $\sim 100$ $150 \mathrm{keV}$ and the upper coronal source up to $\sim 40-80 \mathrm{keV}$ located about $20^{\prime \prime}$ above the LT source, much higher than the energies commonly observed during the impulsive phase when the intense FP emission is also present in the field of view. The opposite spatial gradient of the two coronal sources indicates that electron acceleration is intimately related to the reconnecting current sheet. The spectra from the coronal sources can be described by a power law of a similar index. The high energy LT source exhibits an impulsive temporal profile and soft-hard-soft spectral evolution. The electron density and the percentage of accelerated electrons at the LT source are not well constrained, but a range between $\sim(0.5-5) \times 10^{10} \mathrm{~cm}^{-3}$ and $\sim(1-100) \%$ can be obtained, respectively. These spatial and spectral properties of the coronal sources qualitatively support the stochastic acceleration model. In this scenario, two spatially separated populations of electrons are scattered and accelerated by plasma waves or turbulence below and above the current sheet, generate the two distinct coronal sources by bremsstrahlung, and finally escape from the acceleration regions to the FP re- gions of the flare loop or moving upward in the corona. This latter electron population may further produce the type III radio bursts observed in the flare.

2. The LT and FP spectral difference $(\Delta \gamma \simeq 0-1)$ is found to be much smaller than commonly seen during the impulsive phase. Such a difference should most likely be ascribed to the physical processes in the coronal radiation region. The small difference requires a steeper spectrum of the escaping electrons than that of the accelerated electrons, or an escape time increasing with electron energy. In contrast, stochastic acceleration of electrons by turbulence of the usually assumed Kolmogorov-type spectrum produces an escape time that is very flat or decreases with electron energy. Therefore, more efficient pitch angle scattering and acceleration are required to explain the above observation. More realistic modeling including both kinetic effects and the macroscopic flare structure is needed to address this issue. Inclusion of pitch angle anisotropy of electrons and convergence of magnetic field lines in the corona may help mitigate the above spectral discrepancy.

The above results from imaging spectroscopic analysis of the spatially resolved sources in the 2003 November 3 solar flare highlight the importance of HXR observations with a high dynamic range and sensitivity (e.g. through focusing optics, see Krucker et al. 2011) and over a wide energy range in understanding electron acceleration (and transport) processes.

We thank the RHESSI team for providing the HXR data and the Nançay Radioheliograph team for providing the radio data. We thank the referee Säm Krucker for constructive comments that helped improve the paper. We thank Siming Liu for valuable discussions of the early results, Richard Schwartz, Gordon Hurford, Säm Krucker, David Smith, and Wei Liu for discussions of the pulse pileup effect, Nicole Vilmer and Steven White for discussions of the radio observation, Kim Tolbert, Wei Liu, and Brian Dennis for discussions of OSPEX spectral fitting, Gordon Hurford for discussions of image reconstruction algorithms, Gordon Emslie and Anna Massone for demonstrating the uv_smooth method, and Pascal Saint-Hilaire for discussions of centroid uncertainty. RHESSI is a NASA small explorer mission. This work was supported by NSF SHINE grant ATM0648750 and NASA grant NNX10AC06G.

Facilities: RHESSI.

\section{APPENDIX}

\section{COMPARISON OF IMAGE RECONSTRUCTION ALGORITHMS}

RHESSI is a Fourier imager employing nine rotating modulation collimators to modulate the incident $\mathrm{X}$-ray (and $\gamma$ ray) fluxes from the Sun, which are recorded in nine electrically segmented germanium detectors behind the collimators Lin et al. 2002; Hurford et al. 2002; Smith et al. 2002). HXR images can be reconstructed with several algorithms of general purposes (Hurford et al.|2002), e.g., Clean, MEM_NJIT, uv_smooth, and Pixon.

The Clean algorithm assumes a flare image to be a superposition of point sources, whose location and strength are called the Clean components, and aims to remove the sidelobes of a back projection dirty map (Hurford et al. 2002; Dennis \& Pernak 2009). It iteratively selects the brightest point from the (dirty) residual map and subtract from the map a fraction of its flux multiplied with the point source function (PSF) to form a new residual map until the maximum flux becomes negative or the specified iteration number is reached. In practice, the software convolves these Clean components with the Gaussian-shaped PSF (aka "Clean beam") with addition of the final residual map to display the Clean image. So far Clean is the most commonly used method in the literature for $R H E S S I$ flares; but sometimes the displayed images are rather diffuse. The Pixon method aims to construct the simplest image consistent 

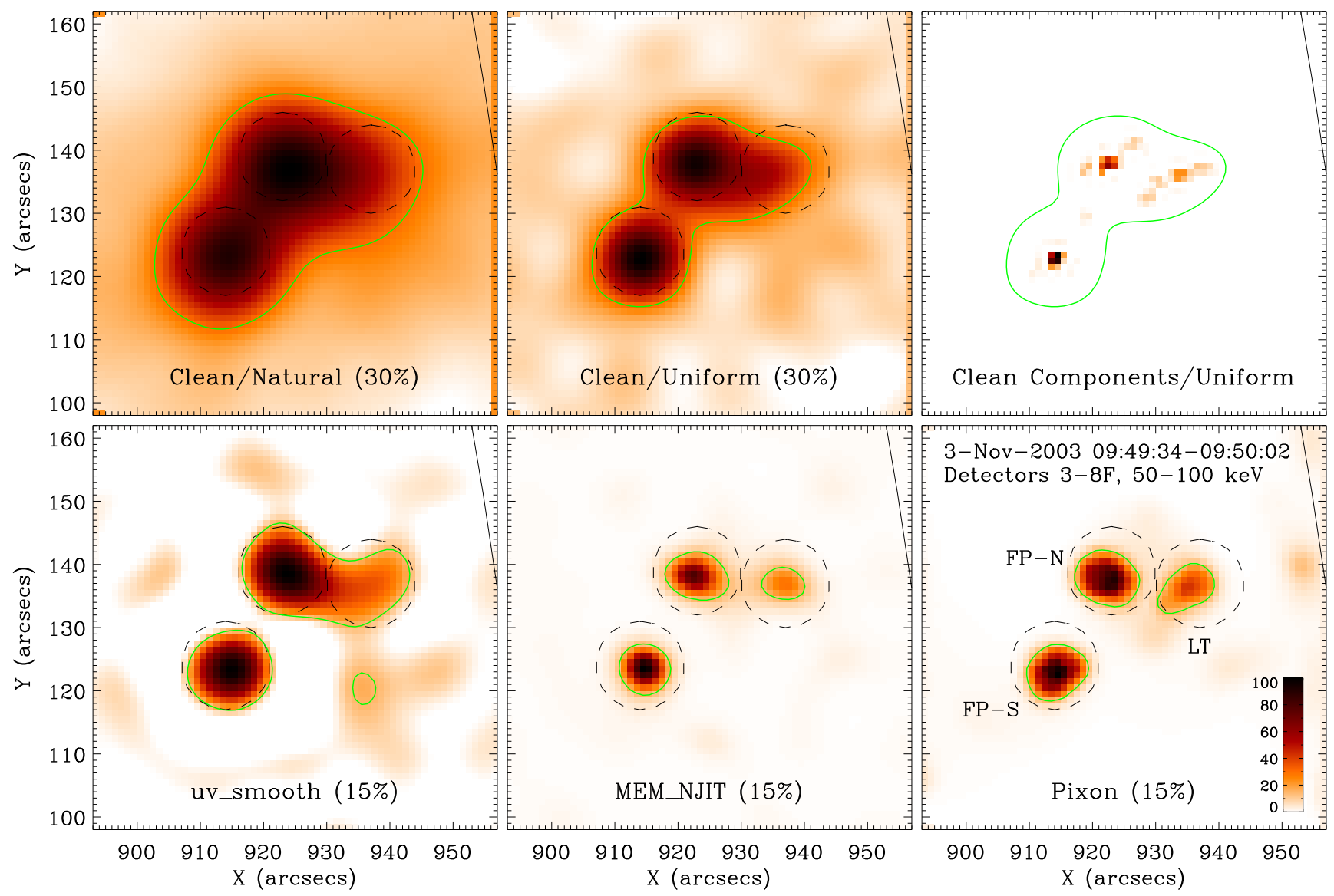

FIG. 10.- HXR images at 50-100 keV during the nonthermal peak as reconstructed by different algorithms from the front segments 3-8. The contours superposed on the images are calculated relative to the maximum intensity of each image. The Clean images show two FP sources as previously found, while the MEM_NJIT and Pixon images, and the Clean components as well, clearly resolve an additional bright LT source.

with the data and is regarded as the most photometrically accurate (Metcalf et al. 1996), although it is much more time-consuming than other methods (Aschwanden et al. 2004). The MEM_NJIT and uv_smooth are algorithms based on the concept of visibility (Hurford et al. 2002), which is the Fourier transforms of the source images, instead of the time-binned modulation profiles for Clean and Pixon. The MEM_NJIT algorithm (Schmahl et al. 2007; Bong et al. 2006) is a fast maximum entropy method (MEM) to maximize the information entropy and the MEM methods usually produce very sharp images, which can help resolve sources close to each other (e.g. Chen \& Ding 2005). The newly invented uv_smooth method (Massone et al. 2009) interpolates a finite set of sparsely sampled visibilities and generate the images through Fourier inversion. More details of these algorithms and comparison among them can be found in Hurford et al. (2002), Aschwanden et al. (2004), Dennis \& Pernak (2009), and Massone et al. (2009).

The 2003 November 3 flare was earlier analyzed with the Clean method (see Liu et al. 2004; Veronig et al. 2006; Ishikawa et al. 2011a). We reanalyze this flare by using all the above methods. In Figure 10 we present the HXR images at 50-100 keV from the front segments 3-8 of FWHM $\sim 6.8^{\prime \prime}$ during the nonthermal peak. It is surprising that the MEM_NJIT and Pixon images show not only two FP sources but also a distinct LT source. Note that actually the Clean components also indicate a rather strong source at the LT location as seen in the Pixon and MEM_NJIT images. On the contrary, in the Clean images, the LT source appears to be part of an apparently elongated source, which was treated as the northern FP. This is due to convolution of the Clean components with the PSF from the segments 3-8, whose default beam width in the software may be too broad to resolve the LT source. The uv_smooth image shows slightly more prominent LT emission than the Clean images, although still much less distinctive than the Pixon and MEM_NJIT images.

We simply ascribe the finding of the high energy LT source to the "super-resolution" power of the Pixon and MEM_NJIT algorithms in resolving close sources. As expected, inclusion of the first segment with the finest angular resolution of FWHM $\sim 2.3^{\prime \prime}$ does reveal the LT source in the Clean images (not shown here).

\section{EXAMINATION OF PULSE PILEUP EFFECT}

Throughout the 2003 November 3 flare, both the thin and thick attenuators (the A3 state) are inserted in place at front of the detectors to reduce the intense thermal emission. The relatively low fractional livetime, $\sim 63 \%$ averaged over the flare nonthermal peak (see also Ishikawa et al. 2011a), indicates potential pulse pileup effect on this X3.9 class 
flare. A preliminary examination (based on the program hsi_pileup_check.pro in SSW) indicates that $\sim 20-30 \%$ of the spatially integrated count rates above $\sim 50 \mathrm{keV}$ are due to pileup. However, We note that this percentage only exceeds the LT contour level at 93-153 keV and is lower than the LT contour levels below $93 \mathrm{keV}$ as shown in Figure 2 .

Pileup occurs when two or more (but with a much lower probability) photons arrive at a detector almost simultaneously such that they are registered indistinguishably as one single photon whose energy is the sum of the individual photon energies (Smith et al. 2002; Hurford et al. 2002). The probability of pileup is roughly proportional to the square of the count rate. Its main effect is to produce spectral distortion, most significantly at energies twice the peak energy of the count rate spectrum $(\sim 18 \mathrm{keV}$ in the A3 state). For very high count rates, it can produce an artificial ("ghost") image at high energy siting atop the main source of the peak energy. We note that, although there exist procedures for preliminary pileup correction of the spatially integrated spectra (Smith et al. 2002) and for forward-modeling simulation in imaging spectroscopy (Schwartz et al. 2008; Liu et al. 2009a), so far the pileup effect and its correction still remain a challenging topic when analyzing imaging spectra.

We show here that the pileup effect should not be important on the distinct, high energy LT coronal source as seen in the current flare. Our confidence relies on the appearance of the high energy LT source itself: its unusually high energies and its large separation from the most intense part of the thermal loop. First, pileup mostly comes from two thermal photons near the peak energy of the count rate spectrum (in first order), therefore the "ghost" image should appear at the same place as the thermal emission (R. A. Schwartz, private communication). Nevertheless, we do not find such a source at energies $\geq 25-50 \mathrm{keV}$. Similarly, Saint-Hilaire et al. (2008) claimed that the pileup effect has negligible influence on the FP spectra for those flares in which the FPs are spatially distinct from the thermal loop. Second, the LT's highest energy is at least five times greater than the peak energy of the count spectrum, which makes it very unlikely for the pileup effect to generate a compact source at such high energies. Note that the pileup effect tends to produce a rather diffuse "ghost" source (G. J. Hurford, private communication). Although pileup in higher orders is more complicated and cannot be completely ignored, its effect on imaging has not been studied so far (S. Krucker, private communication). Third, we note that the upper coronal source that is located away from the thermal loop cannot be due to pileup because of its low thermal intensity. Since bremsstrahlung emissivity is mainly proportional to the local plasma density, in this sense, it is reasonable to expect a more intense LT source at lower altitudes, which is the focus of our study here.

Therefore we believe that the high energy LT source up to $\sim 100-150 \mathrm{keV}$ in the 2003 November 3 flare is physically real and not an artifact of the pileup effect. We cannot find the LT source at higher energies $(\geq 150 \mathrm{keV})$ based on the images reconstructed from the rear segments, which are nearly unaffected by the pileup effect (see also Ishikawa et al. 2011a).

\section{REFERENCES}

Alexander, D., \& Metcalf, T. R. 1997, ApJ, 489, 442

Aschwanden, M. J. 2002, Space Sci. Rev., 101, 1

Aschwanden, M. J., \& Benz, A. O. 1997, ApJ, 480, 825

Aschwanden, M. J., Benz, A. O., Dennis, B. R., \& Schwartz, R. A. 1995, ApJ, 455,347

Aschwanden, M. J., Metcalf, T. R., Krucker, S., Sato, J., Conway, A. J., Hurford, G. J., \& Schmahl, E. J. 2004, Sol. Phys., 219, 149

Bain, H. M., \& Fletcher, L. 2009, A\&A, 508, 1443

Bastian, T. S., Benz, A. O., \& Gary, D. E. 1998, ARA\&A, 36, 131

Battaglia, M., \& Benz, A. O. 2006, A\&A, 456, 751

Battaglia, M., \& Benz, A. O. 2008, A\&A, 487, 337

Bogachev, S. A., Somov, B. V., Kosugi, T., \& Sakao, T. 2005, ApJ, 630, 561

Bong, S.-C., Lee, J., Gary, D. E., \& Yun, H. S. 2006, ApJ, 636, 1159

Brown, J. C. 1971, Sol. Phys., 18, 489

Brown, J. C., Aschwanden, M. J., \& Kontar, E. P. 2002, Sol. Phys., 210, 373

Brown, J. C., Turkmani, R., Kontar, E. P., MacKinnon, A. L., \& Vlahos, L. 2009, A\&A, 508, 993

Bykov, A. M., \& Fleishman, G. D. 2009, ApJ, 692, L45

Chandran, B. D. G. 2003, ApJ, 599, 1426

Chen, Q., \& Petrosian, V. 2009, AGU Fall Meeting Abstracts, A1523

Chen, Q. R., \& Ding, M. D. 2005, ApJ, 618, 537

Christe, S., Krucker, S., \& Lin, R. P. 2008, ApJ, 680, L149

Dauphin, C., Vilmer, N., \& Krucker, S. 2006, A\&A, 455, 339

Dauphin, C., Vilmer, N., Lüthi, T., Trottet, G., Krucker, S., \& Magun, A. 2005, Adv. Space Res., 35, 1805

Dennis, B. R., \& Pernak, R. L. 2009, ApJ, 698, 2131

Emslie, A. G., Kontar, E. P., Krucker, S., \& Lin, R. P. 2003, ApJ, 595, L107

Emslie, A. G., Miller, J. A., \& Brown, J. C. 2004, ApJ, 602, L69

Fletcher, L. 1999, in Proc. 9th European Meeting on Solar Physics, ed. A. Wilson (ESA SP-448; Noordwijk: ESA), 693
Fletcher, L., \& Hudson, H. S. 2008, ApJ, 675, 1645

Fletcher, L., \& Martens, P. C. H. 1998, ApJ, 505, 418

Grigis, P. C., \& Benz, A. O. 2004, A\&A, 426, 1093

Grigis, P. C., \& Benz, A. O. 2006, A\&A, 458, 641

Hannah, I. G., \& Kontar, E. P. 2011, A\&A, 529, A109

Holman, G. D., Kundu, M. R., \& Papadopoulos, K. 1982, ApJ, 257,354

Holman, G. D. 1996, BAAS, 28, 939

Holman, G. D., Sui, L., Schwartz, R. A., \& Emslie, A. G. 2003, ApJ, 595, L97

Hoyng, P., et al. 1981, ApJ, 246, L155

Hudson, H. S. 1972, Sol. Phys., 24, 414

Hudson, H. S., Strong, K. T., Dennis, B. R., Zarro, D., Inda, M., Kosugi, T., \& Sakao, T. 1994, ApJ, 422, L25

Hurford, G. J., et al. 2002, Sol. Phys., 210, 61

Ishikawa, S., Krucker, S., Takahashi, T., \& Lin, R. P. 2011a, ApJ, 728,22

Ishikawa, S., Krucker, S., Takahashi, T., \& Lin, R. P. 2011b, ApJ, 737,48

Jiang, Y. W., Liu, S., Liu, W., \& Petrosian, V. 2006, ApJ, 638, 1140

Kane, S. R. 1981, ApJ, 247, 1113

Kerdraon, A., \& Delouis, J.-M. 1997, in Coronal Physics from Radio and Space Observations, ed. G. Trottet (Berlin: Springer), 192

Krucker, S., et al. 2008a, A\&A Rev., 16, 155

Krucker, S., Christe, S., Glesener, L., et al. 2011, Proc. SPIE, 8147,814705

Krucker, S., Hudson, H. S., Glesener, L., White, S. M., Masuda, S., Wuelser, J.-P., \& Lin, R. P. 2010, ApJ, 714, 1108

Krucker, S., Hurford, G. J., MacKinnon, A. L., Shih, A. Y., \& Lin, R. P. 2008b, ApJ, 678, L63

Krucker, S., \& Lin, R. P. 2008, ApJ, 673, 1181

Krucker, S., Saint-Hilaire, P., Christe, S., White, S. M., Chavier, A. D., Bale, S. D., \& Lin, R. P. 2008c, ApJ, 681, 644

Leach, J. 1984, Ph.D. Thesis, Stanford Univ. 
Leach, J., \& Petrosian, V. 1983, ApJ, 269, 715

Li, Y. P., \& Gan, W. Q. 2007, Adv. Space Res., 39, 1389

Liu, R., Xu, Y., \& Wang, H. 2010, Sol. Phys., 269, 67

Lin, R. P. 1974, Space Sci. Rev., 16, 189

Lin, R. P., et al. 2002, Sol. Phys., 210, 3

Liu, S., \& Fletcher, L. 2009, ApJ, 701, L34

Liu, W. 2006, Ph.D. Thesis, Stanford Univ.

Liu, W., Jiang, Y. W., Liu, S., \& Petrosian, V. 2004, ApJ, 611, L53

Liu, W., Petrosian, V., Dennis, B. R., \& Holman, G. D. 2009a, ApJ, 693, 847

Liu, W., Petrosian, V., Dennis, B. R., \& Jiang, Y. W. 2008, ApJ, 676, 704

Liu, W., Petrosian, V., \& Mariska, J. T. 2009b, ApJ, 702, 1553

Longcope, D. W., \& Guidoni, S. E. 2011, ApJ, 740, 73

Massone, A. M., Emslie, A. G., Hurford, G. J., Prato, M., Kontar, E. P., \& Piana, M. 2009, ApJ, 703, 2004

Masuda, S., Kosugi, T., Hara, H., Sakao, T., Shibata, K., \& Tsuneta, S. 1995, PASJ, 47, 677

Masuda, S., Kosugi, T., Hara, H., Tsuneta, S., \& Ogawara, Y. 1994, Nature, 371, 495

Masuda, S., Sato, J., Kosugi, T., \& Sakao, T. 2000, Adv. Space Res., 26, 493

McTiernan, J. M., Kane, S. R., Loran, J. M., Lemen, J. R., Acton L. W., Hara, H., Tsuneta, S., \& Kosugi, T. 1993, ApJ, 416, L91

Melrose, D. B. \& Brown, J. C. 1976, MNRAS, 176, 15

Metcalf, T. R., \& Alexander, D. 1999, ApJ, 522, 1108

Metcalf, T. R., Hudson, H. S., Kosugi, T., Puetter, R. C., \& Pina, R. K. 1996, ApJ, 466, 585

Miller, J. A., et al. 1997, J. Geophys. Res., 102, 14631

Miller, J. A., Guessoum, N., \& Ramaty, R. 1990, ApJ, 361, 701

Minoshima, T., Masuda, S., Miyoshi, Y., \& Kusano, K. 2011, ApJ, 732, 111

Mrozek, T. 2006, Adv. Space Res., 38, 962

Paesold, G., Benz, A. O., Klein, K.-L., \& Vilmer, N. 2001, A\&A, 371, 333

Petrosian, V. 1973, ApJ, 186, 291
Petrosian, V., \& Chen, Q. 2010, ApJ, 712, L131

Petrosian, V., \& Donaghy, T. Q. 1999, ApJ, 527, 945

Petrosian, V., Donaghy, T. Q., \& McTiernan, J. M. 2002, ApJ, 569, 459

Petrosian, V., \& Liu, S. 2004, ApJ, 610, 550

Priest, E., \& Forbes, T. 2000, Magnetic reconnection: MHD

theory and applications (Cambridge: Cambridge Univ. Press)

Pryadko, J. M., \& Petrosian, V. 1997, ApJ, 482, 774

Pryadko, J. M., \& Petrosian, V. 1998, ApJ, 495, 377

Reid, H. A. S., Vilmer, N., \& Kontar, E. P. 2011, A\&A, 529, A66

Saint-Hilaire, P., Krucker, S., \& Lin, R. P. 2008, Sol. Phys., 250, 53

Sakao, T. 1994, Ph.D. Thesis, Univ. of Tokyo

Schmahl, E. J., Pernak, R. L., Hurford, G. J., Lee, J., \& Bong, S. 2007, Sol. Phys., 240, 241

Schwartz, R. A. 2008, AGU Spring Meeting Abstracts, SP51B-03

Shao, C., \& Huang, G. 2009, ApJ, 691, 299

Smith, D. M., et al. 2002, Sol. Phys., 210, 33

Sturrock, P. A. 1966, Nature, 211, 695

Sui, L., \& Holman, G. D. 2003, ApJ, 596, L251

Sui, L., Holman, G. D., \& Dennis, B. R. 2004, ApJ, 612, 546

Su, Y., Holman, G. D., Dennis, B. R., Tolbert, A. K., \& Schwartz, R. A. 2009, ApJ, 705, 1584

Syrovat-Skii, S. I., \& Shmeleva, O. P. 1972, Soviet Ast., 16, 273

Tomczak, M. 2001, A\&A, 366, 294

Tomczak, M. 2009, A\&A, 502, 665

Veronig, A. M., \& Brown, J. C. 2004, ApJ, 603, L117

Veronig, A. M., Karlický, M., Vršnak, B., Temmer, M., Magdalenić, J., Dennis, B. R., Otruba, W., \& Pötzi, W. 2006, A\&A, 446, 675

Vilmer, N., Krucker, S., Lin, R. P., \& The RHESSI Team 2002, Sol. Phys., 210, 261

Wheatland, M. S., \& Melrose, D. B. 1995, Sol. Phys., 158, 283

Zharkova, V. V., et al. 2011, Space Sci. Rev., in press

Zharkova, V. V., \& Gordovskyy, M. 2006, ApJ, 651, 553 